\title{
On the topological reflexivity of the isometry group of the suspension of $B(H)$
}

\author{
by \\ MÁTÉ GYŐRY (Debrecen)
}

\begin{abstract}
We describe the topological reflexive closure of the isometry group of the suspension of $B(H)$.
\end{abstract}

1. Introduction. The study of reflexive linear subspaces of the algebra $B(H)$ of all bounded linear operators on a Hilbert space $H$ represents one of the most active research areas in operator theory (see [6] for a nice general view of reflexivity of this kind). In the last decades, similar questions concerning certain important sets of transformations acting on Banach algebras rather than on Hilbert spaces have also attracted considerable attention. The initiators of the research in this direction are Kadison, Larson and Sourour. In [10] Kadison studied local derivations from a von Neumann algebra $\mathcal{R}$ into a dual $\mathcal{R}$-bimodule $\mathcal{M}$. He called a continuous linear map from $\mathcal{R}$ into $\mathcal{M}$ a local derivation if it agrees with a derivation at each point in the algebra $\mathcal{R}$ (the derivation may differ from point to point). The main result, Theorem A, in [10] states that in the above setting, every local derivation is a derivation. Independently, Larson and Sourour [12] proved that the same conclusion holds for the local derivations of $B(X)$, where $X$ is a Banach space. Since then, a considerable amount of work has been done concerning local derivations of various algebras (see e.g. $[2,5,7,9,19,20,22-26]$ ).

Besides derivations, there are at least two other important classes of transformations on operator algebras which deserve attention from this point of view, namely, the group of automorphisms and the group of surjective isometries. Larson [11, Some concluding remarks (5), p. 298] initiated the study of local automorphisms (the definition should be self-explanatory) of

2000 Mathematics Subject Classification: Primary 46L40, 47B48, 46E40; Secondary $46 \mathrm{E} 25$

Key words and phrases: reflexivity, automorphisms, surjective isometries, suspension, operator algebras.

This research was supported by the Hungarian National Foundation for Scientific Research (OTKA), Grant No. F038326. 
Banach algebras. In his joint paper with Sourour [12] that we have already mentioned they proved that if $X$ is an infinite-dimensional Banach space, then every surjective local automorphism of $B(X)$ is an automorphism (see also the paper [2] of Brešar and Šemrl). For further results on local automorphisms, we refer to $[3,18,20]$. The common feature of all those results is that they show that the local derivations, local automorphisms, local isometries, etc. of the underlying structures are (global) derivations, automorphisms, isometries, etc., respectively. Clearly, this is a remarkable property of the underlying structure. For function algebras, results of this kind concerning local automorphisms and local isometries were obtained e.g. by Cabello Sánchez and Molnár in [4], and by Molnár and Zalar in [17].

We now define the concept of reflexivity that we shall use. Let $X$ be a Banach space (in fact, in the cases we are interested in, $X$ is usually a Banach algebra) and for any subset $\mathcal{E} \subset B(X)$ let

$$
\begin{aligned}
& \operatorname{ref}_{\text {alg }} \mathcal{E}=\{T \in B(X): T x \in \mathcal{E} x \text { for all } x \in X\}, \\
& \operatorname{ref}_{\text {top }} \mathcal{E}=\{T \in B(X): T x \in \overline{\mathcal{E} x} \text { for all } x \in X\},
\end{aligned}
$$

where the bar denotes norm-closure. The above sets are called the algebraic reflexive closure and the topological reflexive closure of $\mathcal{E}$, respectively. The collection $\mathcal{E}$ of transformations is called algebraically reflexive if $\operatorname{ref}_{\text {alg }} \mathcal{E}=\mathcal{E}$, and topologically reflexive if $\operatorname{ref}_{\text {top }} \mathcal{E}=\mathcal{E}$.

Obviously, topological reflexivity is a stronger property than algebraic reflexivity. Shulman [21] showed that the derivation algebra of any $C^{*}$-algebra is topologically reflexive. For the topological reflexivity of derivation algebras, automorphism groups and isometry groups we refer to $[1,8,13,14]$.

For the automorphism group or the isometry group of $C^{*}$-algebras, a general result as in [21] does not hold. If $X$ is a Banach space, let iso $(X)$ denote the set of all linear (not necessarily surjective) isometries of $X$. The isometry group, i.e. the group of all surjective linear isometries of $X$, is denoted by $\operatorname{Iso}(X)$. If $\mathcal{A}$ is a $*$-algebra then let $\operatorname{Aut}(\mathcal{A})$ and $\operatorname{Aut}^{*}(\mathcal{A})$ denote the group of all automorphisms (i.e. multiplicative linear bijections) and the group of all $*$-automorphisms of $\mathcal{A}$, respectively. Now, if $X$ is an uncountable discrete topological space, then it is not difficult to verify that the groups $\operatorname{Aut}\left(C_{0}(X)\right)$ and $\operatorname{Iso}\left(C_{0}(X)\right)$ of the $C^{*}$-algebra $C_{0}(X)$ of all continuous complex-valued functions on $X$ vanishing at infinity are not reflexive even algebraically. Concerning topological reflexivity, there are even von Neumann algebras whose automorphism and isometry groups are not topologically reflexive. For example, Batty and Molnár [1] showed that the infinite-dimensional commutative von Neumann algebras acting on a separable Hilbert space have this non-reflexivity property.

In the present paper we deal with the reflexivity of the isometry group of the suspension of $B(H)$. The concept of the suspension of a $C^{*}$-algebra plays 
an important role in the $\mathrm{K}$-theory of operator algebras. If $\mathcal{A}$ is a $C^{*}$-algebra then its suspension is the $C^{*}$-tensor product $C_{0}(\mathbb{R}) \otimes \mathcal{A}$, which is well known to be isomorphic to $C_{0}(\mathbb{R}, \mathcal{A})$, the algebra of all continuous functions from $\mathbb{R}$ into $\mathcal{A}$ which vanish at infinity. We know that the automorphism group and the isometry group of $B(H)$ are topologically reflexive if $H$ is a separable infinite-dimensional Hilbert space [13]. In [16] it was shown that $\operatorname{Aut}\left(C_{0}(\mathbb{R})\right)$ and $\operatorname{Iso}\left(C_{0}(\mathbb{R})\right)$ are algebraically (but not topologically) reflexive. The main result in [16] was that the automorphism group and the isometry group of the suspension $C_{0}(\mathbb{R}) \otimes B(H)$ of $B(H)$ are algebraically (but not topologically) reflexive. The referee of that paper raised the interesting problem whether it is possible to describe explicitly the topological reflexive closures of $\operatorname{Aut}\left(C_{0}(\mathbb{R}) \otimes B(H)\right)$ and $\operatorname{Iso}\left(C_{0}(\mathbb{R}) \otimes B(H)\right)$. The present paper gives a solution to this problem.

2. Statement of the results. From now on, let $H$ stand for an infinitedimensional separable Hilbert space. Here we shall describe the elements of the sets $\operatorname{ref}_{\text {top }}\left(\operatorname{Aut}^{*}\left(C_{0}(\mathbb{R}) \otimes B(H)\right)\right)$ and $\operatorname{ref}_{\text {top }}\left(\operatorname{Iso}\left(C_{0}(\mathbb{R}) \otimes B(H)\right)\right)$.

The proof of the main result of this paper is based on the following auxiliary theorem.

Theorem 1. Let $X$ be a Banach space, $\mathcal{S} \subseteq$ iso $(X)$ a topologically reflexive subset and $\phi: C_{0}(\mathbb{R}, X) \rightarrow C_{0}(\mathbb{R}, X)$ a linear map. For any $f \in$ $C_{0}(\mathbb{R}, X)$ there exist homeomorphisms $\varphi_{n}: \mathbb{R} \rightarrow \mathbb{R}(n \in \mathbb{N})$ and functions $\tau_{n}: \mathbb{R} \rightarrow \mathcal{S}(n \in \mathbb{N})$ with

$$
\tau_{n} f \circ \varphi_{n} \rightarrow \phi(f)
$$

if and only if there exists an open interval $U \subseteq \mathbb{R}$, a surjective, monotone, continuous function $\varphi: U \rightarrow \mathbb{R}$, and a function $\tau: U \rightarrow \mathcal{S}$ such that for any $f \in C_{0}(\mathbb{R}, X)$ we have

$$
\phi(f)(y)= \begin{cases}\tau(y)(f(\varphi(y))) & \text { if } y \in U, \\ 0 & \text { if } y \in \mathbb{R} \backslash U .\end{cases}
$$

Moreover, if $\phi$ is of the form (1), then $\tau: U \rightarrow \mathcal{S}$ is strongly continuous.

The *-automorphisms of $C_{0}(\mathbb{R}, B(H))$ are both automorphisms and surjective linear isometries. The paper [16] provides the forms of the surjective linear isometries and the automorphisms of $C_{0}(\mathbb{R}, B(H))$. In view of the topological reflexivity of the isometry group and the automorphism group of $B(H)$ (see [13]), Theorem 1 implies immediately the main result of this paper which reads as follows.

Theorem 2. Let $\phi: C_{0}(\mathbb{R}, B(H)) \rightarrow C_{0}(\mathbb{R}, B(H))$ be a linear map. We have $\phi \in \operatorname{ref}_{\text {top }} \operatorname{Iso}\left(C_{0}(\mathbb{R}, B(H))\right)$, resp. $\phi \in \operatorname{ref}_{\text {top }} \operatorname{Aut}^{*}\left(C_{0}(\mathbb{R}, B(H))\right)$, if and only if there exist an open interval $U \subseteq \mathbb{R}$, a surjective, monotone, 
continuous function $\varphi: U \rightarrow \mathbb{R}$, and $\tau: U \rightarrow \operatorname{Iso}(B(H))$, resp. $\tau: U \rightarrow$ $\operatorname{Aut}^{*}(B(H))$, such that for any $f \in C_{0}(\mathbb{R}, B(H))$, $\phi$ is of the form (1).

Moreover, if $\phi$ is of the form (1), then $\tau$ is strongly continuous.

In [16] it was proved that the isometry group of $C_{0}(\mathbb{R}, B(H))$ is algebraically reflexive. We show that this conclusion can be deduced fairly easily from Theorem 1 as well. We first prove the following auxiliary result, which turns out to be an easy corollary of Theorem 1 .

Theorem 3. Let $X$ be a Banach space, $\mathcal{P} \subseteq$ iso $(X)$ an algebraically reflexive subset and $\phi: C_{0}(\mathbb{R}, X) \rightarrow C_{0}(\mathbb{R}, X)$ a linear map. If for any $f \in C_{0}(\mathbb{R}, X)$ there exist a homeomorphism $\varphi_{f}: \mathbb{R} \rightarrow \mathbb{R}$ and a function $\tau_{f}: \mathbb{R} \rightarrow \mathcal{P}$ such that

$$
\phi(f)=\tau_{f} f \circ \varphi_{f},
$$

then there exist a homeomorphism $\varphi: \mathbb{R} \rightarrow \mathbb{R}$ and a function $\tau: \mathbb{R} \rightarrow \mathcal{P}$ such that for any $f \in C_{0}(\mathbb{R}, X)$ we have

$$
\phi(f)=\tau f \circ \varphi,
$$

and in this case $\tau: \mathbb{R} \rightarrow \mathcal{P}$ is strongly continuous.

Now we immediately obtain the following theorem, which was the main result of $[16]$.

Theorem 4. The groups $\operatorname{Iso}\left(C_{0}(\mathbb{R}, B(H))\right)$ and Aut* $\left(C_{0}(\mathbb{R}, B(H))\right)$ are algebraically reflexive.

\section{Proofs}

Proof of Theorem 1. First suppose that $\phi$ is of the form (1), where $U=$ ]$u_{1}, u_{2}\left[\right.$ with $u_{1}, u_{2} \in \mathbb{R} \cup\{-\infty, \infty\}, \varphi: U \rightarrow \mathbb{R}$ and $\tau: U \rightarrow \mathcal{S}$ are as in Theorem 1. Extend $\tau: U \rightarrow \mathcal{S}$ to a function $\tau: \mathbb{R} \rightarrow \mathcal{S}$. Let $u_{1}<a_{n} \in U$ and $u_{2}>b_{n} \in U$ be real sequences with $a_{n} \rightarrow u_{1}$ and $b_{n} \rightarrow u_{2}$. Now for any $n \in \mathbb{N}$ there exists a homeomorphism $\varphi_{n}: \mathbb{R} \rightarrow \mathbb{R}$ for which $\left|\varphi(y)-\varphi_{n}(y)\right|<1 / n$ for any $y \in\left[a_{n}, b_{n}\right]$, and $\varphi\left(\left[a_{n}, b_{n}\right]\right) \subseteq \varphi_{n}\left(\left[a_{n}, b_{n}\right]\right)$.

We show that for any $f \in C_{0}(\mathbb{R}, X)$,

$$
\tau f \circ \varphi_{n} \rightarrow \phi(f) .
$$

Let $\varepsilon>0$. Then there exists a compact set $K \subseteq \mathbb{R}$ such that $\|f(y)\|<\varepsilon / 2$ $(y \in \mathbb{R} \backslash K)$. Since $a_{n} \rightarrow u_{1}, b_{n} \rightarrow u_{2}$ and $\varphi: U \rightarrow \mathbb{R}$ is surjective, there exists $n_{1} \in \mathbb{N}$ such that $K \subseteq \varphi\left(\left[a_{n}, b_{n}\right]\right)^{\circ}$ for any $n \geq n_{1}$. Since $f \in C_{0}(\mathbb{R}, X)$ is uniformly continuous, there exists $\delta>0$ such that for any $x, y \in K,|x-y|<\delta$ implies $\|f(x)-f(y)\|<\varepsilon$. Let $n_{2} \in \mathbb{N}$ with $1 / n_{2}<\delta$ and $n_{0}=\max \left(n_{1}, n_{2}\right)$. Further, let $y \in U$ and $n \geq n_{0}$. If $y \in\left[a_{n}, b_{n}\right]$ then $\left|\varphi(y)-\varphi_{n}(y)\right|<1 / n<\delta$, and thus $\left\|f(\varphi(y))-f\left(\varphi_{n}(y)\right)\right\|<\varepsilon$. If $y \in U \backslash\left[a_{n}, b_{n}\right]$ then, by the monotonicity of $\varphi_{n}$ and $\varphi$, we have $\varphi_{n}(y) \notin \varphi_{n}\left(\left[a_{n}, b_{n}\right]\right)^{\circ} \supseteq \varphi\left(\left[a_{n}, b_{n}\right]\right)^{\circ} \supseteq K$ 
and $\varphi(y) \notin \varphi\left(\left[a_{n}, b_{n}\right]\right)^{\circ} \supseteq K$, so $\|f(\varphi(y))\|,\left\|f\left(\varphi_{n}(y)\right)\right\|<\varepsilon / 2$, which implies $\left\|f(\varphi(y))-f\left(\varphi_{n}(y)\right)\right\|<\varepsilon$. Then, as $\tau(y) \in \operatorname{iso}(X)$, for any $y \in U$ and $n \geq n_{0}$ we have

$$
\begin{aligned}
\left\|\left(\tau f \circ \varphi_{n}\right)(y)-\phi(f)(y)\right\| & =\left\|\tau(y) f\left(\varphi_{n}(y)\right)-\tau(y) f(\varphi(y))\right\| \\
& =\left\|f\left(\varphi_{n}(y)\right)-f(\varphi(y))\right\|<\varepsilon .
\end{aligned}
$$

For any $y \in \mathbb{R} \backslash U$ we obtain $y \notin\left[a_{n}, b_{n}\right]$, thus $\varphi_{n}(y) \notin \varphi_{n}\left(\left[a_{n}, b_{n}\right]\right)^{\circ} \supseteq$ $\varphi\left(\left[a_{n}, b_{n}\right]\right)^{\circ} \supseteq K$, which implies

$$
\left\|\left(\tau f \circ \varphi_{n}\right)(y)-\phi(f)(y)\right\|=\left\|\left(\tau f \circ \varphi_{n}\right)(y)\right\|=\left\|f\left(\varphi_{n}(y)\right)\right\|<\varepsilon / 2 .
$$

Hence $\tau f \circ \varphi_{n} \rightarrow \tau f \circ \varphi=\phi(f)$, which completes the proof in one direction.

Consider now the other direction. Suppose that for any $f \in C_{0}(\mathbb{R}, X)$ there are homeomorphisms $\varphi_{f, n}: \mathbb{R} \rightarrow \mathbb{R}(n \in \mathbb{N})$ and functions $\tau_{f, n}: \mathbb{R} \rightarrow \mathcal{S}$ $(n \in \mathbb{N})$ such that

$$
\tau_{f, n} f \circ \varphi_{f, n} \rightarrow \phi(f) .
$$

In what follows, for brevity, $\varphi_{f, n}$ and $\tau_{f, n}$ will denote the functions corresponding to $f \in C_{0}(\mathbb{R}, X)$. Now $\phi$ is obviously an isometry. For any $x \in \mathbb{R}$ and $A \in X$ with $A \neq 0$, set

$$
\begin{gathered}
S_{0}(x, A)=\left\{f \in C_{0}(\mathbb{R}, X):\|f(x)\|=\|f\|>0, f(x) /\|f(x)\|=A /\|A\|,\right. \\
\forall y \in \mathbb{R}, y \neq x:\|f(y)\|<\|f\|\}, \\
S(x, A)=\left\{f \in C_{0}(\mathbb{R}, X):\|f(x)\|=\|f\|>0, f(x) /\|f(x)\|=A /\|A\|\right\},
\end{gathered}
$$

which are clearly non-empty sets.

Real sequences tending to $-\infty$ or $+\infty$ will be considered convergent. Further, for any $f \in C_{0}(\mathbb{R}, X)$ define $f(-\infty)=0$ and $f(+\infty)=0$.

SteP 1 . For any $x \in \mathbb{R}$ and $A \in X$ with $A \neq 0$, the set

$$
G(x, A)=\bigcap_{f \in S(x, A)}\{y \in \mathbb{R}:\|\phi(f)(y)\|=\|f\|\}
$$

is non-empty and compact.

Let $f \in S(x, A)$. As $\phi(f) \in C_{0}(\mathbb{R}, X)$, the set $\{y \in \mathbb{R}:\|\phi(f)(y)\|=\|f\|\}$ is compact, so to prove that $G(x, A) \neq \emptyset$, it is sufficient to show that the system of these sets has the finite intersection property.

Let $n \in \mathbb{N}$ and $f_{1}, \ldots, f_{n} \in S(x, A)$. Then

$$
\begin{aligned}
\left\|f_{1}+\cdots+f_{n}\right\| & \geq\left\|\left(f_{1}+\cdots+f_{n}\right)(x)\right\|=\left\|f_{1}(x)+\cdots+f_{n}(x)\right\| \\
& =\left\|\frac{\left\|f_{1}(x)\right\|}{\|A\|} \cdot A+\cdots+\frac{\left\|f_{n}(x)\right\|}{\|A\|} \cdot A\right\| \\
& =\left\|\left(\left\|f_{1}\right\|+\cdots+\left\|f_{n}\right\|\right) \frac{1}{\|A\|} \cdot A\right\|=\left\|f_{1}\right\|+\cdots+\left\|f_{n}\right\|,
\end{aligned}
$$


thus

$$
\left\|f_{1}+\cdots+f_{n}\right\|=\left\|f_{1}\right\|+\cdots+\left\|f_{n}\right\| .
$$

Since $\phi\left(f_{1}+\cdots+f_{n}\right) \in C_{0}(\mathbb{R}, X)$, there exists $z \in \mathbb{R}$ for which $\| \phi\left(f_{1}+\ldots\right.$ $\left.+f_{n}\right)(z)\|=\| \phi\left(f_{1}+\cdots+f_{n}\right) \|$. Hence

$$
\begin{aligned}
\left\|\phi\left(f_{1}\right)(z)\right\|+\ldots+\left\|\phi\left(f_{n}\right)(z)\right\| & \geq\left\|\phi\left(f_{1}+\ldots+f_{n}\right)(z)\right\|=\left\|\phi\left(f_{1}+\ldots+f_{n}\right)\right\| \\
& =\left\|f_{1}+\ldots+f_{n}\right\|=\left\|f_{1}\right\|+\ldots+\left\|f_{n}\right\| \\
& =\left\|\phi\left(f_{1}\right)\right\|+\ldots+\left\|\phi\left(f_{n}\right)\right\| \\
& \geq\left\|\phi\left(f_{1}\right)(z)\right\|+\ldots+\left\|\phi\left(f_{n}\right)(z)\right\|,
\end{aligned}
$$

which implies

$$
\left\|\phi\left(f_{i}\right)(z)\right\|=\left\|\phi\left(f_{i}\right)\right\|=\left\|f_{i}\right\| \quad(1 \leq i \leq n),
$$

thus

$$
z \in \bigcap_{i=1}^{n}\left\{y \in \mathbb{R}:\left\|\phi\left(f_{i}\right)(y)\right\|=\left\|f_{i}\right\|\right\} .
$$

Consequently, $G(x, A)$ is indeed a non-empty compact set.

We note that for any $x \in \mathbb{R}, A \in X$ with $A \neq 0$ and $\lambda>0$, we obviously have

$$
G(x, \lambda A)=G(x, A) .
$$

STEP 2. If $x \in \mathbb{R}, A \in X, A \neq 0, f \in C_{0}(\mathbb{R}, X)$ and

$$
f(x)=(\|f(x)\| /\|A\|) A
$$

then

$$
\|\phi(f)(y)\| \geq\|f(x)\| \quad(y \in G(x, A)) .
$$

By (4), we may assume that $\|A\|=1$. Let $y \in G(x, A)$. If $\|f(x)\|=\|f\|$ then by Step 1 we are done. So we may assume that $\|f(x)\|<\|f\|$. Now it is easy to verify that there exists an $f_{0} \in S(x, A)$ such that $\left\|f_{0}\right\|=$ $\|f\|-\|f(x)\|>0, f+f_{0} \in S(x, A)$ and $\left\|f+f_{0}\right\|=\|f\|$. Let $h=f+f_{0}$. By Step 1, we have

$$
\|\phi(h)(y)\|=\|h\|=\|f\|,
$$

which implies

$$
\begin{aligned}
\|f\| & =\|\phi(h)(y)\|=\left\|\phi(f)(y)+\phi\left(f_{0}\right)(y)\right\| \\
& \leq\|\phi(f)(y)\|+\left\|\phi\left(f_{0}\right)\right\|=\|\phi(f)(y)\|+(\|f\|-\|f(x)\|),
\end{aligned}
$$

thus $\|\phi(f)(y)\| \geq\|f(x)\|$.

Step 3. For any $x \in \mathbb{R}, A \in X$ with $A \neq 0$, and any $f \in S_{0}(x, A)$, the set

$$
\{y \in \mathbb{R}:\|\phi(f)(y)\|=\|f\|\}
$$

is a compact interval which contains $G(x, A)$. 
Let $f \in S_{0}(x, A)$, and set $y_{1}=\inf \{y \in \mathbb{R}:\|\phi(f)(y)\|=\|f\|\}, \quad y_{2}=\sup \{y \in \mathbb{R}:\|\phi(f)(y)\|=\|f\|\}$. Then

$$
\lim _{n \rightarrow \infty}\left\|f\left(\varphi_{f, n}\left(y_{1}\right)\right)\right\|=\|f\|, \quad \lim _{n \rightarrow \infty}\left\|f\left(\varphi_{f, n}\left(y_{2}\right)\right)\right\|=\|f\| .
$$

Let $u_{n}$ be a subsequence of $\varphi_{f, n}\left(y_{1}\right)$ or $\varphi_{f, n}\left(y_{2}\right)$. By (5) and since $0 \neq$ $f \in C_{0}(\mathbb{R}, X)$, the sequence $u_{n}$ has an accumulation point $y_{0} \in \mathbb{R}$. Then (5) implies $\left\|f\left(y_{0}\right)\right\|=\|f\|$. As $f \in S_{0}(x, A)$, we have $y_{0}=x$. So every subsequence of $\varphi_{f, n}\left(y_{1}\right)$ or $\varphi_{f, n}\left(y_{2}\right)$ has a subsequence converging to $x$, which implies

$$
\lim _{n \rightarrow \infty} \varphi_{f, n}\left(y_{1}\right)=\lim _{n \rightarrow \infty} \varphi_{f, n}\left(y_{2}\right)=x .
$$

Let $y \in\left[y_{1}, y_{2}\right]$. Since $\varphi_{f, n}: \mathbb{R} \rightarrow \mathbb{R}(n \in \mathbb{N})$ is a homeomorphism (which is clearly monotone), from (6) and $y_{1} \leq y \leq y_{2}$ we have $\varphi_{f, n}(y) \rightarrow x$, thus $\|\phi(f)(y)\|=\lim _{n \rightarrow \infty}\left\|\tau_{f, n}(y) f\left(\varphi_{f, n}(y)\right)\right\|=\lim _{n \rightarrow \infty}\left\|f\left(\varphi_{f, n}(y)\right)\right\|=\|f(x)\|=\|f\|$. Hence $\{y \in \mathbb{R}:\|\phi(f)(y)\|=\|f\|\}=\left[y_{1}, y_{2}\right]$, and we are done.

SteP 4. If $x \in \mathbb{R}$ and $A \in X$ with $A \neq 0$, then $G(x, A) \subset \mathbb{R}$ is a compact interval.

Let $y \in[\inf G(x, A), \sup G(x, A)]$ and $f \in S(x, A)$. Then there exist functions $f_{n} \in S_{0}(x, A)(n \in \mathbb{N})$ with $f_{n} \rightarrow f$. For any $f_{n} \in S_{0}(x, A)$ we have $G(x, A) \subseteq\left\{z \in \mathbb{R}:\left\|\phi\left(f_{n}\right)(z)\right\|=\left\|f_{n}\right\|\right\}$, thus, by Step 3, we obtain $[\inf G(x, A), \sup G(x, A)] \subseteq\left\{z \in \mathbb{R}:\left\|\phi\left(f_{n}\right)(z)\right\|=\left\|f_{n}\right\|\right\}$. Hence $\|\phi(f)(y)\|=\lim _{n \rightarrow \infty}\left\|\phi\left(f_{n}\right)(y)\right\|=\lim _{n \rightarrow \infty}\left\|f_{n}\right\|=\|f\|$. Thus $y \in G(x, A)$, and we are done.

SteP 5. Let $f \in C_{0}(\mathbb{R}, X)$ and suppose there exist $\left.\varepsilon \in\right] 0,1 / 100[$, elements $A_{1}, A_{2}, A_{3} \in X$ of norm 1 , real numbers $p<x_{1}<z_{1}<x_{2}<$ $z_{2}<x_{3}<z_{3}<x_{4}$ and disjoint closed intervals $J_{0}<I_{1}<J_{1}<I_{2}<$ $J_{2}<I_{3}<J_{3}<I_{4}$ with $\left.\left.J_{0}=\right]-\infty, p\right], x_{i} \in I_{i}(1 \leq i \leq 4), z_{i} \in J_{i}$ $(1 \leq i \leq 3), f\left(x_{1}\right)=A_{1}, f\left(x_{2}\right)=A_{2}, f\left(x_{3}\right)=A_{3},\left\|f\left(x_{4}\right)\right\|=1 / 2, f\left(z_{1}\right)=0$, $\left\|f\left(z_{2}\right)\right\|<\varepsilon,\left\|f\left(z_{3}\right)\right\|<\varepsilon,\|f\|=1$, and with

$$
\begin{aligned}
& \|f(x)\| \\
& \in \begin{cases}{[0,4 \varepsilon]} & \text { if } x \in J_{0} \cup J_{1} \cup J_{2} \cup J_{3}, \\
] 4 \varepsilon, 1-4 \varepsilon[ & \text { if } x \in] \sup J_{0}, \inf J_{3}\left[\backslash\left(I_{1} \cup J_{1} \cup I_{2} \cup J_{2} \cup I_{3}\right),\right. \\
{[1-4 \varepsilon, 1]} & \text { if } x \in I_{1} \cup I_{2} \cup I_{3}, \\
] 4 \varepsilon, 1 / 2-4 \varepsilon[ & \text { if } \sup J_{3}<x<\inf I_{4}, \\
{[1 / 2-4 \varepsilon, 1 / 2]} & \text { if } x \in I_{4}, \\
{[0,1 / 2-4 \varepsilon[} & \text { if } \sup I_{4}<x .\end{cases}
\end{aligned}
$$


Then there is $y \in\left[\inf G\left(x_{1}, A_{1}\right), \sup G\left(x_{3}, A_{3}\right)\right] \cup\left[\inf G\left(x_{3}, A_{3}\right), \sup G\left(x_{1}, A_{1}\right)\right]$ such that $\phi(f)(y)=0$. Moreover,

$G\left(x_{1}, A_{1}\right)<G\left(x_{2}, A_{2}\right)<G\left(x_{3}, A_{3}\right) \quad$ or $\quad G\left(x_{1}, A_{1}\right)>G\left(x_{2}, A_{2}\right)>G\left(x_{3}, A_{3}\right)$.

\section{Let}

$K_{1}=\{y \in \mathbb{R}: \nexists a, b \in \mathbb{R}: a<b<y,\|\phi(f)(a)\| \geq 1 / 2-2 \varepsilon,\|\phi(f)(b)\| \leq 2 \varepsilon$,

$\exists a, b \in \mathbb{R}: y<a<b,\|\phi(f)(a)\| \leq 2 \varepsilon,\|\phi(f)(b)\| \geq 1-2 \varepsilon\}$,

$K_{1}^{\prime}=\{y \in \mathbb{R}: \nexists a, b \in \mathbb{R}: a<b<y,\|\phi(f)(a)\| \geq 1-2 \varepsilon,\|\phi(f)(b)\| \leq 2 \varepsilon$,

$\exists a, b \in \mathbb{R}: a<b<y,\|\phi(f)(a)\| \geq 1 / 2-2 \varepsilon,\|\phi(f)(b)\| \leq 2 \varepsilon$,

$\exists a, b \in \mathbb{R}: y<a<b,\|\phi(f)(a)\| \leq 2 \varepsilon,\|\phi(f)(b)\| \geq 1-2 \varepsilon\}$,

$K_{2}=\left\{y \in \mathbb{R}: \exists a_{1}, a_{2}, b_{1}, b_{2} \in \mathbb{R}: a_{1}<a_{2}<y<b_{1}<b_{2}\right.$,

$\left.\left\|\phi(f)\left(a_{1}\right)\right\|,\left\|\phi(f)\left(b_{2}\right)\right\| \geq 1-2 \varepsilon,\left\|\phi(f)\left(a_{2}\right)\right\|,\left\|\phi(f)\left(b_{1}\right)\right\| \leq 2 \varepsilon\right\}$,

$K_{3}=\{y \in \mathbb{R}: \nexists a, b \in \mathbb{R}: y<a<b,\|\phi(f)(a)\| \leq 2 \varepsilon,\|\phi(f)(b)\| \geq 1 / 2-2 \varepsilon$,

$\exists a, b \in \mathbb{R}: a<b<y,\|\phi(f)(a)\| \geq 1-2 \varepsilon,\|\phi(f)(b)\| \leq 2 \varepsilon\}$,

$K_{3}^{\prime}=\{y \in \mathbb{R}: \nexists a, b \in \mathbb{R}: y<a<b,\|\phi(f)(a)\| \leq 2 \varepsilon,\|\phi(f)(b)\| \geq 1-2 \varepsilon$,

$\exists a, b \in \mathbb{R}: y<a<b,\|\phi(f)(a)\| \leq 2 \varepsilon,\|\phi(f)(b)\| \geq 1 / 2-2 \varepsilon$,

$\exists a, b \in \mathbb{R}: a<b<y,\|\phi(f)(a)\| \geq 1-2 \varepsilon,\|\phi(f)(b)\| \leq 2 \varepsilon\}$.

It is easy to see that $K_{1}, K_{1}^{\prime}, K_{2}, K_{3}$ and $K_{3}^{\prime}$ are pairwise disjoint intervals. Let $f_{1} \in S_{0}\left(x_{1}, A_{1}\right), f_{2} \in S_{0}\left(x_{2}, A_{2}\right), f_{3} \in S_{0}\left(x_{3}, A_{3}\right)$ be functions with disjoint supports for which $\left\|f_{1}\right\|,\left\|f_{2}\right\|,\left\|f_{3}\right\|=\varepsilon$ and $f_{i}\left(z_{j}\right)=0(i=1,2,3$; $j=1,2)$. Then $f+f_{2} \in S_{0}\left(x_{2}, A_{2}\right)$ and $\left\|f+f_{2}\right\|=1+\varepsilon$. We may assume that there are $y_{1}, y_{2}, y_{3}, u_{1}, u_{2} \in \mathbb{R} \cup\{+\infty,-\infty\}$ for which

$$
\begin{array}{ll}
\varphi_{f+f_{2}, n}^{-1}\left(x_{1}\right) \rightarrow y_{1}, & \varphi_{f+f_{2}, n}^{-1}\left(x_{2}\right) \rightarrow y_{2}, \quad \varphi_{f+f_{2}, n}^{-1}\left(x_{3}\right) \rightarrow y_{3}, \\
\varphi_{f+f_{2}, n}^{-1}\left(z_{1}\right) \rightarrow u_{1}, \quad \varphi_{f+f_{2}, n}^{-1}\left(z_{2}\right) \rightarrow u_{2} .
\end{array}
$$

Then $\tau_{f+f_{2}, n} \cdot\left(f+f_{2}\right) \circ \varphi_{f+f_{2}, n} \rightarrow \phi\left(f+f_{2}\right)$ implies

$$
\begin{aligned}
\left\|\phi\left(f+f_{2}\right)\left(y_{1}\right)\right\| & =\left\|\left(f+f_{2}\right)\left(x_{1}\right)\right\|=1, \\
\left\|\phi\left(f+f_{2}\right)\left(u_{1}\right)\right\| & =\left\|\left(f+f_{2}\right)\left(z_{1}\right)\right\|=0, \\
\left\|\phi\left(f+f_{2}\right)\left(y_{2}\right)\right\| & =\left\|\left(f+f_{2}\right)\left(x_{2}\right)\right\|=1+\varepsilon, \\
\left\|\phi\left(f+f_{2}\right)\left(u_{2}\right)\right\| & =\left\|\left(f+f_{2}\right)\left(z_{2}\right)\right\|<\varepsilon, \\
\left\|\phi\left(f+f_{2}\right)\left(y_{3}\right)\right\| & =\left\|\left(f+f_{2}\right)\left(x_{3}\right)\right\|=1 .
\end{aligned}
$$

Thus $y_{1}, y_{2}, y_{3} \in \mathbb{R}$. By the monotonicity of $\varphi_{f+f_{2}, n}(n \in \mathbb{N})$, we have $y_{1}<$ $u_{1}<y_{2}<u_{2}<y_{3}$ or $y_{3}<u_{2}<y_{2}<u_{1}<y_{1}$. Hence (8) and $\left\|\phi\left(f_{2}\right)\right\|=$ $\left\|f_{2}\right\|=\varepsilon$ imply 


$$
\begin{aligned}
\left\|\phi(f)\left(y_{1}\right)\right\| & \geq 1-\varepsilon, & \left\|\phi(f)\left(y_{2}\right)\right\| & \geq 1, \quad\left\|\phi(f)\left(y_{3}\right)\right\| \geq 1-\varepsilon, \\
\left\|\phi(f)\left(u_{1}\right)\right\| & \leq \varepsilon, & \left\|\phi(f)\left(u_{2}\right)\right\| & \leq 2 \varepsilon .
\end{aligned}
$$

Thus $y_{2} \in K_{2}$. Now since $f+f_{2} \in S_{0}\left(x_{2}, A_{2}\right)$, Step 3 implies that $\{y \in \mathbb{R}$ : $\left.\left\|\phi\left(f+f_{2}\right)(y)\right\|=\left\|f+f_{2}\right\|=1+\varepsilon\right\}$ is a compact interval which contains $y_{2}$. Then, by the definition of $K_{2}, y_{2} \in K_{2}$ clearly yields

$$
\left\{y \in \mathbb{R}:\left\|\phi\left(f+f_{2}\right)(y)\right\|=\left\|f+f_{2}\right\|=1+\varepsilon\right\} \subseteq K_{2},
$$

which implies

$$
G\left(x_{2}, A_{2}\right) \subseteq K_{2}
$$

We have $f+f_{1} \in S_{0}\left(x_{1}, A_{1}\right)$ and $\left\|f+f_{1}\right\|=1+\varepsilon$. We may assume that there are $y_{1}, y_{2}, u_{1} \in \mathbb{R} \cup\{+\infty,-\infty\}$ such that

$$
\varphi_{f+f_{1}, n}^{-1}\left(x_{1}\right) \rightarrow y_{1}, \quad \varphi_{f+f_{1}, n}^{-1}\left(x_{2}\right) \rightarrow y_{2}, \quad \varphi_{f+f_{1}, n}^{-1}\left(z_{1}\right) \rightarrow u_{1} .
$$

Now, as above, we deduce that

$$
\begin{aligned}
\left\|\phi\left(f+f_{1}\right)\left(y_{1}\right)\right\| & =\left\|\left(f+f_{1}\right)\left(x_{1}\right)\right\|=1+\varepsilon, \\
\left\|\phi\left(f+f_{1}\right)\left(u_{1}\right)\right\| & =\left\|\left(f+f_{1}\right)\left(z_{1}\right)\right\|=0, \\
\left\|\phi\left(f+f_{1}\right)\left(y_{2}\right)\right\| & =\left\|\left(f+f_{1}\right)\left(x_{2}\right)\right\|=1,
\end{aligned}
$$

and either $y_{1}<u_{1}<y_{2}$ or $y_{2}<u_{1}<y_{1}$. Hence, by (10), we have $y_{1}, y_{2}, u_{2} \in \mathbb{R}$. Again, as above, since $\left\|\phi\left(f_{1}\right)\right\|=\left\|f_{1}\right\|=\varepsilon$, (10) implies

$$
\left\|\phi(f)\left(y_{1}\right)\right\| \geq 1, \quad\left\|\phi(f)\left(u_{1}\right)\right\| \leq \varepsilon, \quad\left\|\phi(f)\left(y_{2}\right)\right\| \geq 1-\varepsilon .
$$

Suppose that $y_{1}<u_{1}<y_{2}$. Further, suppose on the contrary that there are $a, b \in \mathbb{R}$ such that $a<b<y_{1},\|\phi(f)(a)\| \geq 1 / 2-2 \varepsilon$ and $\|\phi(f)(b)\| \leq 2 \varepsilon$. We may assume that there exist $u_{a}, u_{b} \in \mathbb{R} \cup\{+\infty,-\infty\}$ for which

$$
\varphi_{f+f_{1}, n}(a) \rightarrow u_{a}, \quad \varphi_{f+f_{1}, n}(b) \rightarrow u_{b} .
$$

Then $\left\|f_{1}\right\|=\varepsilon$ implies

$$
\begin{aligned}
\left\|\left(f+f_{1}\right)\left(u_{a}\right)\right\| & =\left\|\phi\left(f+f_{1}\right)(a)\right\| \geq\left|\|\phi(f)(a)\|-\left\|\phi\left(f_{1}\right)(a)\right\|\right| \\
& \geq 1 / 2-2 \varepsilon-\varepsilon=1 / 2-3 \varepsilon \\
\left\|\left(f+f_{1}\right)\left(u_{b}\right)\right\| & =\left\|\phi\left(f+f_{1}\right)(b)\right\| \leq\|\phi(f)(b)\|+\left\|\phi\left(f_{1}\right)(b)\right\| \leq 2 \varepsilon+\varepsilon=3 \varepsilon,
\end{aligned}
$$

thus

$$
\left\|f\left(u_{a}\right)\right\| \geq 1 / 2-3 \varepsilon-\varepsilon=1 / 2-4 \varepsilon, \quad\left\|f\left(u_{b}\right)\right\| \leq 3 \varepsilon+\varepsilon=4 \varepsilon .
$$

By (9) and since $a<b<y_{1}<y_{2}$, except for a finite number of $n \in \mathbb{N}$, we have $a<b<\varphi_{f+f_{1}, n}^{-1}\left(x_{1}\right)<\varphi_{f+f_{1}, n}^{-1}\left(x_{2}\right)$, which implies that $\varphi_{f+f_{1}, n}^{-1}$ is increasing. Thus $u_{a} \leq u_{b} \leq x_{1}$, which contradicts (12), (7) and $x_{1} \in I_{1}$. This means that there do not exist $a, b \in \mathbb{R}$ such that $a<b<y_{1},\|\phi(f)(a)\| \geq$ $1 / 2-2 \varepsilon$ and $\|\phi(f)(b)\| \leq 2 \varepsilon$. Hence $(11)$ and $y_{1}<u_{1}<y_{2}$ imply $y_{1} \in K_{1}$. Similarly, if $y_{2}<u_{1}<y_{1}$ then $y_{1} \in K_{3}$. Consequently, $y_{1} \in K_{1}$ or $y_{1} \in K_{3}$. Since $f+f_{1} \in S_{0}\left(x_{1}, A_{1}\right)$, by Step 3 , the set $\left\{y \in \mathbb{R}:\left\|\phi\left(f+f_{1}\right)(y)\right\|=\right.$ 
$\left.\left\|f+f_{1}\right\|=1+\varepsilon\right\}$ is a compact interval which contains $y_{1}$. By the definitions of $K_{1}$ and $K_{3}$, it is now clear that either

$$
\left\{y \in \mathbb{R}:\left\|\phi\left(f+f_{1}\right)(y)\right\|=\left\|f+f_{1}\right\|=1+\varepsilon\right\} \subseteq K_{1} \text { or } K_{3},
$$

which implies

$$
G\left(x_{1}, A_{1}\right) \subseteq K_{1} \quad \text { or } \quad G\left(x_{1}, A_{1}\right) \subseteq K_{3} .
$$

In a similar way it can be proved that

$$
G\left(x_{3}, A_{3}\right) \subseteq K_{1}^{\prime} \quad \text { or } \quad G\left(x_{3}, A_{3}\right) \subseteq K_{3}^{\prime} .
$$

Now let $v_{1} \in G\left(x_{1}, A_{1}\right)$ and $v_{3} \in G\left(x_{3}, A_{3}\right)$. Suppose on the contrary that $G\left(x_{1}, A_{1}\right) \subseteq K_{1}$ and $G\left(x_{3}, A_{3}\right) \subseteq K_{1}^{\prime}$. Then $v_{1} \in K_{1}, v_{3} \in K_{1}^{\prime}$, and

$$
\left\|\phi(f)\left(v_{1}\right)\right\|=\left\|\phi(f)\left(v_{3}\right)\right\|=1 .
$$

Suppose that $v_{1}<v_{3}$. Then, as $v_{3} \in K_{1}^{\prime}$, there exist $a<b<v_{3}$ for which $\|\phi(f)(a)\| \geq 1 / 2-2 \varepsilon$ and $\|\phi(f)(b)\| \leq 2 \varepsilon$. Since $\left\|\phi(f)\left(v_{1}\right)\right\|=1$, the inequality $v_{1}<b<v_{3}$ would contradict $v_{3} \in K_{1}^{\prime}$. Thus $a<b<v_{1}$, hence $\|\phi(f)(a)\| \geq 1 / 2-2 \varepsilon$ and $\|\phi(f)(b)\| \leq 2 \varepsilon$ contradicts $v_{1} \in K_{1}$. Similarly we get a contradiction in the case $v_{3}<v_{1}$. Thus $G\left(x_{1}, A_{1}\right) \subseteq K_{1}$ and $G\left(x_{3}, A_{3}\right) \subseteq K_{1}^{\prime}$ cannot hold simultaneously. Similarly, $G\left(x_{1}, A_{1}\right) \subseteq K_{3}$ and $G\left(x_{3}, A_{3}\right) \subseteq K_{3}^{\prime}$ cannot both hold. Thus

$$
G\left(x_{1}, A_{1}\right) \subseteq K_{1}, G\left(x_{3}, A_{3}\right) \subseteq K_{3}^{\prime} \quad \text { or } \quad G\left(x_{1}, A_{1}\right) \subseteq K_{3}, G\left(x_{3}, A_{3}\right) \subseteq K_{1}^{\prime} .
$$

It is easy to see that $K_{1}<K_{2}<K_{3}^{\prime}$ and $K_{1}^{\prime}<K_{2}<K_{3}$, whence $G\left(x_{1}, A_{1}\right)<G\left(x_{2}, A_{2}\right)<G\left(x_{3}, A_{3}\right) \quad$ or $\quad G\left(x_{1}, A_{1}\right)>G\left(x_{2}, A_{2}\right)>G\left(x_{3}, A_{3}\right)$.

Finally, we may assume that there are $y_{1}, y_{2}, y_{3}, u_{1}, u_{2} \in \mathbb{R} \cup\{+\infty,-\infty\}$ with

$$
\varphi_{f, n}^{-1}\left(x_{i}\right) \rightarrow y_{i}(1 \leq i \leq 3), \quad \varphi_{f, n}^{-1}\left(z_{j}\right) \rightarrow u_{j}(1 \leq j \leq 2) .
$$

Now since $\tau_{f, n} \cdot f \circ \varphi_{f, n} \rightarrow \phi(f)$, the monotonicity of $\varphi_{f, n}(n \in \mathbb{N})$ implies $\left\|\phi(f)\left(y_{1}\right)\right\|=\left\|f\left(x_{1}\right)\right\|=1,\left\|\phi(f)\left(y_{2}\right)\right\|=\left\|f\left(x_{2}\right)\right\|=1,\left\|\phi(f)\left(u_{1}\right)\right\|=$ $\left\|f\left(z_{1}\right)\right\|=0$ and $y_{1}<u_{1}<y_{2}$ or $y_{2}<u_{1}<y_{1}$. Then $y_{1}, y_{2} \in \mathbb{R}$, and so $u_{1} \in \mathbb{R}$. Moreover, $u_{1} \in K_{2}$. Then, since $K_{1}<K_{2}<K_{3}^{\prime}$ and $K_{1}^{\prime}<K_{2}<K_{3}$, we are done.

STEP 6. For any $A, B \in X$ with $A, B \neq 0$, and for $x, y \in \mathbb{R}$ with $x \neq y$, we have

$$
G(x, A) \cap G(y, B)=\emptyset .
$$

Moreover, $G$ is "monotone" in the sense that either

$$
\begin{array}{ll}
G\left(x_{1}, A\right)<G\left(x_{2}, B\right) & \text { for every } x_{1}, x_{2} \in \mathbb{R} \text { with } x_{1}<x_{2}, \text { or } \\
G\left(x_{1}, A\right)>G\left(x_{2}, B\right) & \text { for every } x_{1}, x_{2} \in \mathbb{R} \text { with } x_{1}<x_{2}
\end{array}
$$

where the relations " $<$ " and " $>$ " are understood pointwise. 
Let $x_{1}, x_{2}, x_{3}$ and $A_{1}, A_{2}, A_{3} \in X$ satisfy $x_{1}<x_{2}<x_{3}$ and $\left\|A_{1}\right\|=$ $\left\|A_{2}\right\|=\left\|A_{3}\right\|=1$. It is easy to see that there exists a function $f \in C_{0}(\mathbb{R}, X)$ such that $f$ satisfies the conditions of Step 5. Then, by Step 5, we are done.

STEP 7. $G$ is "continuous" in the following sense: for any $x_{n} \rightarrow x_{0}$ and $A \in X$ we have $G\left(x_{n}, A\right) \rightarrow G\left(x_{0}, A\right)$, i.e.

$$
\sup \left\{d\left(y, G\left(x_{0}, A\right)\right): y \in G\left(x_{n}, A\right)\right\} \rightarrow 0,
$$

where

$$
d\left(y, G\left(x_{0}, A\right)\right)=\inf \left\{|y-z|: z \in G\left(x_{0}, A\right)\right\} .
$$

Let $x_{n} \in \mathbb{R}$ be a decreasing sequence with $x_{n} \rightarrow x_{0} \in \mathbb{R}$. (If $x_{n}$ is increasing then the proof is similar.) For simplicity, assume that $G$ is increasing. Suppose

$$
y_{0}=\lim _{n \rightarrow \infty} \sup G\left(x_{n}, A\right) \geq \sup G\left(x_{0}, A\right)
$$

and let $f \in S\left(x_{0}, A\right)$. Then there exist $f_{n} \in S\left(x_{n}, A\right)(n \in \mathbb{N})$ for which $\left\|f_{n}\right\|=\|f\|(n \in \mathbb{N})$ and $f_{n} \rightarrow f$. Since $\sup G\left(x_{n}, A\right) \rightarrow y_{0}$ and $\phi(f)$ is continuous, we have

$$
\left\|\phi(f)\left(y_{0}\right)\right\|=\lim _{n \rightarrow \infty}\left\|\phi\left(f_{n}\right)\left(\sup G\left(x_{n}, A\right)\right)\right\|=\lim _{n \rightarrow \infty}\left\|f_{n}\right\|=\|f\| .
$$

Hence $y_{0} \in G\left(x_{0}, A\right)$, thus $y_{0}=\sup G\left(x_{0}, A\right)$. Since $G$ is monotone, we are done.

STEP 8. Let $G(x)=G(x, I)$ for any $x \in \mathbb{R}$, where $I \in X$ is fixed. Then for any $A \in X$ with $A \neq 0$, we have

$$
G(x)=G(x, A) .
$$

Let $A, B \in X$ with $A, B \neq 0$, and let $x_{n}$ be a decreasing sequence with $x<x_{n} \rightarrow x$. For simplicity, assume that $G$ is increasing. Then Steps 6 and 7 imply that

$$
\sup G(x, A)<\inf G\left(x_{n}, B\right) \rightarrow \sup G(x, B),
$$

thus

$$
\sup G(x, A) \leq \sup G(x, B) .
$$

In a similar way we get $\sup G(x, B) \leq \sup G(x, A)$, thus $\sup G(x, A)=$ $\sup G(x, B)$. Similarly $\inf G(x, A)=\inf G(x, B)$, and, by Step 4, we are done.

STEP 9. Let

$$
U=\bigcup_{x \in \mathbb{R}} G(x) .
$$

Then $U$ is an open interval. For any $u \in U$ denote by $\varphi(u) \in \mathbb{R}$ the uniquely determined real number for which $u \in G(\varphi(u))$. Then $\varphi: U \rightarrow \mathbb{R}$ is surjective, continuous and monotone. Moreover,

$$
\|\phi(f)(y)\| \geq\|f(\varphi(y))\| \quad(y \in U) .
$$


By Steps 2 and 8 and the definition of $\varphi$, we immediately get (13).

The definition of $\varphi$ and Step 6 imply that $\varphi$ is monotone. It is clear that $\varphi$ is surjective. We show that $\varphi$ is also continuous. Let $u_{n} \in U(n \in \mathbb{N})$ and $u \in U$ be such that $u_{n} \rightarrow u$. Further, let

$$
x_{1}=\liminf _{n \rightarrow \infty} \varphi\left(u_{n}\right), \quad x_{2}=\limsup _{n \rightarrow \infty} \varphi\left(u_{n}\right) .
$$

Then $x_{1}, x_{2} \in \mathbb{R}$, and there exists a subsequence $v_{n}$ of $u_{n}$ for which

$$
x_{1}=\lim _{n \rightarrow \infty} \varphi\left(v_{n}\right) .
$$

Then, by Step 7 and the definition of $\varphi$, we have

$$
v_{n} \in G\left(\varphi\left(v_{n}\right)\right) \rightarrow G\left(x_{1}\right),
$$

whence

$$
u=\lim _{n \rightarrow \infty} v_{n} \in G\left(x_{1}\right)
$$

Similarly

$$
u \in G\left(x_{2}\right) .
$$

Hence $x_{1}=x_{2}=\varphi(u)$, so $\varphi\left(u_{n}\right) \rightarrow \varphi(u)$. Thus $\varphi$ is indeed continuous.

We now show that $U$ is an interval. Suppose on the contrary that there exist $a_{0}, b_{0} \in U$ and $z \in \mathbb{R} \backslash U$ such that $a_{0}<z<b_{0}$. Let

$$
z_{1}=\sup (]-\infty, z[\cap U), \quad z_{2}=\inf (] z, \infty[\cap U) .
$$

There exists an increasing sequence $\left.a_{n} \in\right]-\infty, z[\cap U(n \in \mathbb{N})$ and a decreasing sequence $\left.b_{n} \in\right] z, \infty\left[\cap U(n \in \mathbb{N})\right.$ such that $a_{n} \rightarrow z_{1}$ and $b_{n} \rightarrow z_{2}$. Since $\varphi$ is monotone and $a_{1} \leq a_{n}<z<b_{n} \leq b_{1}(n \in \mathbb{N})$, there exist $a, b \in \mathbb{R}$ for which $\varphi\left(a_{n}\right) \rightarrow a$ and $\varphi\left(b_{n}\right) \rightarrow b$. By the "continuity" of $G$, we have $a_{n} \in G\left(\varphi\left(a_{n}\right)\right) \rightarrow G(a)$ and $b_{n} \in G\left(\varphi\left(b_{n}\right)\right) \rightarrow G(b)$, from which we obtain

$$
z_{1}=\lim _{n \rightarrow \infty} a_{n} \in G(a), \quad z_{2}=\lim _{n \rightarrow \infty} a_{n} \in G(b) .
$$

If $a \neq b$ then there is a $\left.z_{0} \in\right] a, b\left[\right.$, thus $G(a)<G\left(z_{0}\right)<G(b)$ or $G(b)<$ $G\left(z_{0}\right)<G(a)$, whence $\left.U \supseteq G\left(z_{0}\right) \subseteq\right] z_{1}, z_{2}[$. So $U \cap] z_{1}, z_{2}[\neq \emptyset$, which is a contradiction. Hence $a=b$, which implies $z_{1}, z_{2} \in G(a)$. Therefore $z \in\left[z_{1}, z_{2}\right] \subseteq G(a) \subseteq U$. This is again a contradiction, so $U$ is an interval indeed. Hence, by the definition of $U$ and the monotonicity of $G$, we conclude that $U$ is an open interval, which completes the proof of Step 9 .

STEP 10. Let $f \in C_{0}(\mathbb{R}, X)$ and $z_{1} \in \mathbb{R}$ be such that $f$ satisfies the conditions of Step 5 and $f^{-1}(0)=\left\{z_{1}\right\}$. Then there exists $y \in G\left(z_{1}\right)$ for which

$$
\phi(f)(y)=f\left(z_{1}\right)=0 .
$$

By Steps 5 and 9 , there exists $y \in U$ such that $\phi(f)(y)=0$. Then Step 2 implies $\|f(\varphi(y))\| \leq\|\phi(f)(y)\|=0$. Since $f^{-1}(0)=\left\{z_{1}\right\}$, we obtain $\varphi(y)=z_{1}$, thus $y \in G\left(z_{1}\right)$ and $\phi(f)(y)=f\left(z_{1}\right)=0$. 
STEP 11. Let $f \in C_{0}(\mathbb{R}, X)$ and $z_{1} \in \mathbb{R}$ be such that $f$ satisfies the conditions of Step 5 and $f\left(z_{1}\right)=0$. Then for any $y \in G\left(z_{1}\right)$ we have

$$
\phi(f)(y)=f\left(z_{1}\right)=0 .
$$

Let $a=\inf G\left(z_{1}\right), b=\sup G\left(z_{1}\right)$, and let $x \in[a, b]$ and $\left.\varepsilon \in\right] 0, \varepsilon_{0}[$. By the continuity of $f$, there is a $\delta>0$ such that for any $y \in \mathbb{R}$ with $\left|y-z_{1}\right| \leq \delta$ we have $\|f(y)\|<\varepsilon_{0}$. Let $z_{a}, z_{b} \in \mathbb{R}$ with $z_{1}-\delta<z_{a}<z_{1}<z_{b}<z+\delta$. Then there exists $f_{0} \in C_{0}(\mathbb{R}, X)$ such that $\left\|f_{0}\right\|<\varepsilon_{0}$ and $f_{1}=f-f_{0}$ vanishes exactly at $z_{a}, z_{b}$ and $z_{1}$. It is easy to see that there are $g_{n} \in C_{0}(\mathbb{R}, X)$ $(n \in \mathbb{N})$ which satisfy the conditions of Step 5 and for which $g_{n} \rightarrow f_{1}$ and $g_{n}^{-1}(0)=\left\{z_{1}\right\}(n \in \mathbb{N})$. There are $h_{n, a} \in C_{0}(\mathbb{R}, X)(n \in \mathbb{N})$ which satisfy the conditions of Step 5 with $z_{a}$ instead of $z_{1}$ and for which $h_{n, a} \rightarrow f_{1}$ and $h_{n, a}^{-1}(0)=\left\{z_{a}\right\}(n \in \mathbb{N})$. Similarly, there are $h_{n, b} \in C_{0}(\mathbb{R}, X)(n \in \mathbb{N})$ which satisfy the conditions of Step 5 with $z_{b}$ instead of $z_{1}$ and for which $h_{n, b} \rightarrow f_{1}$ and $h_{n, b}^{-1}(0)=\left\{z_{b}\right\}(n \in \mathbb{N})$. Then, by Step 10, for any $n \in \mathbb{N}$ there are $r_{n} \in G\left(z_{1}\right), s_{n, a} \in G\left(z_{a}\right)$ and $s_{n, b} \in G\left(z_{b}\right)$ such that $\phi\left(g_{n}\right)\left(r_{n}\right)=0$, $\phi\left(h_{n, a}\right)\left(s_{n, a}\right)=0$ and $\phi\left(h_{n, b}\right)\left(s_{n, b}\right)=0$. Since $G\left(z_{1}\right), G\left(z_{a}\right)$ and $G\left(z_{b}\right)$ are compact intervals, we may assume that there are $y_{1} \in G\left(z_{1}\right), y_{a} \in G\left(z_{a}\right)$ and $y_{b} \in G\left(z_{b}\right)$ for which $r_{n} \rightarrow y_{1}, s_{n, a} \rightarrow y_{a}$ and $s_{n, b} \rightarrow y_{b}$. Since $g_{n} \rightarrow$ $f_{1}, h_{n, a} \rightarrow f_{1}$ and $h_{n, b} \rightarrow f_{1}$, we deduce that $\phi\left(f_{1}\right)\left(y_{1}\right)=\phi\left(f_{1}\right)\left(y_{a}\right)=$ $\phi\left(f_{1}\right)\left(y_{b}\right)=0$. For simplicity, assume that $G$ is increasing. Then $y_{a}<a \leq$ $y_{1} \leq b<y_{b}$.

We may assume that there are $u_{a}, u, u_{b} \in \mathbb{R} \cup\{+\infty,-\infty\}$ such that $\varphi_{f_{1}, n}\left(y_{a}\right) \rightarrow u_{a}, \varphi_{f_{1}, n}(x) \rightarrow u$ and $\varphi_{f_{1}, n}\left(y_{b}\right) \rightarrow u_{b}$. Now $\left\|f_{1}\left(\varphi_{f_{1}, n}\left(y_{a}\right)\right)\right\| \rightarrow$ $\left\|\phi\left(f_{1}\right)\left(y_{a}\right)\right\|=0$ and $\left\|f_{1}\left(\varphi_{f_{1}, n}\left(y_{b}\right)\right)\right\| \rightarrow\left\|\phi\left(f_{1}\right)\left(y_{b}\right)\right\|=0$. Hence $f_{1}\left(u_{a}\right)=$ $f_{1}\left(u_{b}\right)=0$, which implies $u_{a}, u_{b} \in\left\{z_{a}, z_{1}, z_{b}\right\} \subseteq\left[z_{a}, z_{b}\right]$. Then since $y_{a}<$ $x<y_{b}$, the monotonicity of $\varphi_{f_{1}, n}(n \in \mathbb{N})$ implies $u \in\left[u_{a}, u_{b}\right] \subseteq\left[z_{a}, z_{b}\right] \subseteq$ $\left[z_{1}-\delta, z_{1}+\delta\right]$, thus $\|f(u)\|<\varepsilon_{0}$, and so $\left\|f_{1}(u)\right\|<2 \varepsilon_{0}$. Hence $f_{1}\left(\varphi_{f_{1}, n}(x)\right) \rightarrow$ $f_{1}(u)$ and $\left\|f_{1}\left(\varphi_{f_{1}, n}(x)\right)\right\| \rightarrow\left\|\phi\left(f_{1}\right)(x)\right\|$ imply $\left\|\phi\left(f_{1}\right)(x)\right\|<2 \varepsilon_{0}$, from which we infer that $\|\phi(f)(x)\|<3 \varepsilon_{0}$. Since $\left.\varepsilon_{0} \in\right] 0, \varepsilon[$ is arbitrary, we obtain $\phi(f)(x)=0$. Thus $\phi(f)$ is 0 on the interval $[a, b]=G\left(z_{1}\right)$, as claimed.

SteP 12. Let $f \in C_{0}(\mathbb{R}, X)$ and $x \in \mathbb{R}$. Then for any $y \in G(x)$ we have

$$
\|\phi(f)(y)\|=\|f(x)\| .
$$

It is clear that there exists a function $f_{0} \in C_{0}(\mathbb{R}, X)$ such that $\left(f-f_{0}\right)(x)=0$ and $\left\|f_{0}\right\|=\left\|f_{0}(x)\right\|=\|f(x)\|$. Let $g=f-f_{0}$. It is not difficult to see that there exist $n \in \mathbb{N}, \lambda_{i} \in \mathbb{R}$ and $f_{n} \in C_{0}(\mathbb{R}, X)(1 \leq i \leq n)$ such that $g=\lambda_{1} f_{1}+\cdots+\lambda_{n} f_{n}$, where the functions $f_{1}, \ldots, f_{n}$ satisfy the conditions of Step 5 with $z_{1}=x$. By Step 11, for any $y \in G(x)=G\left(z_{1}\right)$ we have $\phi\left(f_{i}\right)(y)=0$, whence $\phi(g)(y)=\sum_{i=1}^{n} \lambda_{i} \phi\left(f_{i}\right)(y)=0$, and thus $\|\phi(f)(y)\|=\left\|\phi\left(f_{0}\right)(y)\right\|=\left\|f_{0}(x)\right\|=\|f(x)\|$. 
SteP 13. For any $y \in \mathbb{R} \backslash U$ we have $\phi(f)(y)=0$.

Let $u_{1}=\inf U, u_{2}=\sup U$, and let $f \in C_{0}(\mathbb{R}, X)$ be nowhere vanishing. First let $y \in \mathbb{R}$ be such that $\|f(y)\|=\|f\|$ and let $x \in G(y)$. Then $\|f(\varphi(x))\|=\|f(y)\|=\|f\|$. By Step 12, we easily obtain $\phi(f)\left(u_{1}\right)=$ $\phi(f)\left(u_{2}\right)=0$. Thus $\left\|f\left(\varphi_{f, n}\left(u_{1}\right)\right)\right\| \rightarrow 0$ and $\left\|f\left(\varphi_{f, n}\left(u_{2}\right)\right)\right\| \rightarrow 0$, hence $f(y) \neq 0(y \in \mathbb{R})$ implies

$$
\left|\varphi_{f, n}\left(u_{1}\right)\right| \rightarrow \infty, \quad\left|\varphi_{f, n}\left(u_{2}\right)\right| \rightarrow \infty .
$$

If $\varphi_{f, n}\left(u_{1}\right) \rightarrow \infty$ and $\varphi_{f, n}\left(u_{2}\right) \rightarrow \infty$, then since $u_{1}<x<u_{2}$, the monotonicity of $\varphi_{f, n}(n \in \mathbb{N})$ implies $\varphi_{f, n}(x) \rightarrow \infty$, from which we deduce that $\phi(f)(x)=0$. Then $0=\|\phi(f)(x)\|=\|f(\varphi(x))\|=\|f\|>0$, which is a contradiction. Similarly, we obtain a contradiction if $\varphi_{f, n}\left(u_{1}\right) \rightarrow-\infty$ and $\varphi_{f, n}\left(u_{2}\right) \rightarrow-\infty$. Thus either $\varphi_{f, n}\left(u_{1}\right) \rightarrow-\infty$ and $\varphi_{f, n}\left(u_{2}\right) \rightarrow \infty \quad$ or $\quad \varphi_{f, n}\left(u_{2}\right) \rightarrow-\infty$ and $\varphi_{f, n}\left(u_{1}\right) \rightarrow \infty$. Now let $y \in \mathbb{R} \backslash] u_{1}, u_{2}\left[\right.$. Then the monotonicity of $\varphi_{f, n}(n \in \mathbb{N})$ implies $\left.\varphi_{f, n}(y) \notin\right] \varphi_{f, n}\left(u_{1}\right), \varphi_{f, n}\left(u_{2}\right)\left[\right.$. Hence $\left|\varphi_{f, n}(y)\right| \rightarrow \infty$, and so

$$
\|\phi(f)(y)\|=\lim _{n \rightarrow \infty}\left\|f\left(\varphi_{f, n}(y)\right)\right\|=0 .
$$

Now let $f \in C_{0}(\mathbb{R}, X)$ be arbitrary. Then there exist nowhere vanishing functions $f_{n} \in C_{0}(\mathbb{R}, X)(n \in \mathbb{N})$ with $f_{n} \rightarrow f$. It follows from the above that

$$
\phi(f)(y)=\lim _{n \rightarrow \infty} \phi\left(f_{n}\right)(y)=0 .
$$

As $y \in \mathbb{R} \backslash U$ is arbitrary, the proof of Step 13 is complete.

STEP 14. For any $f \in C_{0}(\mathbb{R}, X)$, we have

$$
\|\phi(f)(y)\|= \begin{cases}\|f(\varphi(y))\| & \text { if } y \in U, \\ 0 & \text { if } y \in \mathbb{R} \backslash U .\end{cases}
$$

This is a consequence of Steps 9, 12 and 13. that

STEP 15. There exists a strongly continuous function $\tau: U \rightarrow \mathcal{S}$ such

$$
\phi(f)(y)=\tau(y)(f(\varphi(y))) \quad(y \in U) .
$$

Let $y \in U$ and $A \in X$. Further, let $f \in C_{0}(\mathbb{R}, X)$ with $f(\varphi(y))=A$, and let

$$
\tau(y)(A)=\phi(f)(y)
$$

We show that $\tau(y)$ is well defined. Let $f_{1}, f_{2} \in C_{0}(\mathbb{R}, X)$ for which $f_{1}(\varphi(y))=$ $f_{2}(\varphi(y))=A$. Then, by Step 14 , we have $\left\|\phi\left(f_{1}-f_{2}\right)(y)\right\|=\left\|\left(f_{1}-f_{2}\right)(\varphi(y))\right\|$ $=0$, thus $\phi\left(f_{1}\right)(y)=\phi\left(f_{2}\right)(y)$. Now $\tau(y): X \rightarrow X$ is clearly a linear isometry. 
Let $y \in U$ and $A \in X$ with $A \neq 0$. Further, let $f \in S_{0}(\varphi(y), A)$ be such that $f(\varphi(y))=A$ and $f(x)=(\|f(x)\| /\|A\|) A(x \in \mathbb{R})$. Since $\tau_{f, n} f \circ \varphi_{f, n}$ $\rightarrow \phi(f)$, we have

$$
\tau_{f, n}(y) f\left(\varphi_{f, n}(y)\right) \rightarrow \phi(f)(y)=\tau(y)(A),
$$

thus

$$
\tau_{f, n}(y)\left(\frac{\left\|f\left(\varphi_{f, n}(y)\right)\right\|}{\|A\|} \cdot A\right) \rightarrow \tau(y)(A) .
$$

Since $\tau_{f, n}(y)$ and $\tau(y)$ are isometries, we have $\left\|f\left(\varphi_{f, n}(y)\right)\right\| \rightarrow\|A\|$ and so

$$
\tau_{f, n}(y)(A) \rightarrow \tau(y)(A) .
$$

By the topological reflexivity of $\mathcal{S}$, we obtain $\tau(y) \in \mathcal{S}$.

We now show that $\tau$ is strongly continuous. Let $A \in X, x \in \mathbb{R}$ and $x_{n} \in \mathbb{R}(n \in \mathbb{N})$ with $A \neq 0$ and $x_{n} \rightarrow x$. Then the continuity of $\varphi$ implies $\varphi\left(x_{n}\right) \rightarrow \varphi(x) \in \mathbb{R}$, thus there exists $f \in C_{0}(\mathbb{R}, X)$ for which $f\left(\varphi\left(x_{n}\right)\right)=$ $f(\varphi(x))=A(n \in \mathbb{N})$. Then the continuity of $\phi(f)$ yields

$$
\tau\left(x_{n}\right)(A)=\tau\left(x_{n}\right)\left(f\left(\varphi\left(x_{n}\right)\right)\right)=\phi(f)\left(x_{n}\right) \rightarrow \phi(f)(x)=\tau(x)(A) .
$$

Theorem 1 is now a consequence of Steps 9, 13 and 15.

Proof of Theorem 3. Assume that $\phi$ satisfies the conditions of Theorem 3, and let $\mathcal{S}=\operatorname{iso}(X)$. Then $\phi$ also satisfies the conditions of Theorem 1. Thus, by Theorem 1 , there is an open interval $U \subseteq \mathbb{R}$, a monotone, continuous, surjective function $\varphi: U \rightarrow \mathbb{R}$, and a strongly continuous function $\tau$ : $U \rightarrow \operatorname{iso}(X)$ such that for any $f \in C_{0}(\mathbb{R}, X)$ the equation (1) holds. Let $x \in \mathbb{R}$, and let $f \in C_{0}(\mathbb{R}, X)$ be a nowhere vanishing function for which $\{x\}=\{y \in \mathbb{R}:\|f(y)\|=\|f\|\}$. Since $\phi$ satisfies the conditions of Theorem 3, $\phi(f)$ vanishes nowhere and $\{y \in \mathbb{R}:\|\phi(f)(y)\|=\|\phi(f)\|=\|f(x)\|\}$ is a singleton. By (1), $U=\mathbb{R}$ and $\varphi^{-1}(x)$ is also a singleton. Hence $\varphi$ is injective. Thus $\varphi: \mathbb{R} \rightarrow \mathbb{R}$ is a continuous bijection, and so it is a homeomorphism.

Now let $y \in \mathbb{R}$ and $A \in B(X)$, and let $f \in S_{0}(\varphi(y), A)$ with $f(\varphi(y))=A$. By the conditions of Theorem 3, there is a homeomorphism $\varphi_{0}: \mathbb{R} \rightarrow \mathbb{R}$ and a function $\tau_{0}: \mathbb{R} \rightarrow \mathcal{P}$ for which (2) holds. Then

$$
\|f(\varphi(y))\|=\|\phi(f)(y)\|=\left\|f\left(\varphi_{0}(y)\right)\right\|,
$$

which together with $f \in S_{0}(\varphi(y), A)$ imply $\varphi(y)=\varphi_{0}(y)$. Thus

$$
\begin{aligned}
\tau(y)(A) & =\tau(y)(f(\varphi(y)))=\phi(f)(y) \\
& =\tau_{0}(y)\left(f\left(\varphi_{0}(y)\right)\right)=\tau_{0}(y)(f(\varphi(y)))=\tau_{0}(y)(A) .
\end{aligned}
$$

Now the algebraic reflexivity of $\mathcal{P}$ implies $\tau(y) \in \mathcal{P}$, which completes the proof. 


\section{References}

[1] C. J. K. Batty and L. Molnár, On topological reflexivity of the groups of *-automorphisms and surjective isometries of B(H), Arch. Math. (Basel) 67 (1996), 415-421.

[2] M. Brešar and P. Šemrl, Mappings which preserve idempotents, local automorphisms, and local derivations, Canad. J. Math. 45 (1993), 483-496.

[3] -, -, On local automorphisms and mappings that preserve idempotents, Studia Math. 113 (1995), 101-108.

[4] F. Cabello Sánchez and L. Molnár, Reflexivity of the isometry group of some classical spaces, Rev. Mat. Iberoamericana 18 (2002), 409-430.

[5] R. L. Crist, Local derivations on operator algebras, J. Funct. Anal. 135 (1996), $76-92$.

[6] D. Hadwin, A general view of reflexivity, Trans. Amer. Math. Soc. 344 (1994), $325-360$.

[7] D. Han and S. Wei, Local derivations of nest algebras, Proc. Amer. Math. Soc. 123 (1995), 3095-3100.

[8] W. Jing, S. J. Lu, and G. G. Han, On topological reflexivity of the spaces of derivations on operator algebras, Appl. Math. J. Chinese Univ. Ser. B 17 (2002), 75-79.

[9] B. E. Johnson, Local derivations on $C^{*}$-algebras are derivations, Trans. Amer. Math. Soc. 353 (2001), 313-325.

[10] R. V. Kadison, Local derivations, J. Algebra 130 (1990), 494-509.

[11] D. R. Larson, Reflexivity, algebraic reflexivity and linear interpolation, Amer. J. Math. 110 (1988), 283-299.

[12] D. R. Larson and A. R. Sourour, Local derivations and local automorphisms of $B(X)$, in: Proc. Sympos. Pure Math. 51, Amer. Math. Soc., 1990, 187-194.

[13] L. Molnár, The set of automorphisms of $B(H)$ is topologically reflexive in $B(B(H))$, Studia Math. 122 (1997), 183-193.

[14] - A proper standard $C^{*}$-algebra whose automorphism and isometry groups are topologically reflexive, Publ. Math. Debrecen 52 (1998), 563-574.

[15] - Reflexivity of the automorphism and isometry groups of $C^{*}$-algebras in BDF theory, Arch. Math. (Basel) 74 (2000), 120-128.

[16] L. Molnár and M. Györy, Reflexivity of the automorphism and isometry groups of the suspension of $B(H)$, J. Funct. Anal. 159 (1998), 568-586.

[17] L. Molnár and B. Zalar, Reflexivity of the group of surjective isometries of some Banach spaces, Proc. Edinburgh Math. Soc. 42 (1999), 17-36.

[18] - - - On local automorphisms of group algebras of compact groups, Proc. Amer. Math. Soc. 128 (2000), 93-99.

[19] A. Nowicki, On local derivations in the Kadison sense, Colloq. Math. 89 (2001), 193-198.

[20] E. Scholz and W. Timmermann, Local derivations, automorphisms and commutativity preserving maps on $L^{+}(D)$, Publ. Res. Inst. Math. Sci. 29 (1993), 977-995.

[21] V. S. Shulman, Operators preserving ideals in $C^{*}$-algebras, Studia Math. 109 (1994), $67-72$.

[22] M. Wiehl, Local derivations on the Weyl algebra with one pair of generators, Acta Math. Hungar. 92 (2001), 51-59.

[23] J. Wu, Local derivations of reflexive algebras, Proc. Amer. Math. Soc. 125 (1997), 869-873.

[24] -, Local derivations of reflexive algebras II, ibid. 129 (2001), 1733-1737.

[25] Y. H. Yon, Local derivations of the polynomial ring over a field, Bull. Korean Math. Soc. 36 (1999), 247-257. 
[26] J. Zhu, Local derivations of nest algebras, Proc. Amer. Math. Soc. 123 (1995), 739-742.

Institute of Mathematics

University of Debrecen

P.O.Box 12

4010 Debrecen, Hungary

E-mail: gyorym@math.klte.hu

Received March 9, 2004

Revised version August 20, 2004 